\title{
Regularization of the linearized gravitational self-force for branes
}

\author{
Richard A. Battye, ${ }^{1}$ Brandon Carter, ${ }^{2}$ and Andrew Mennim ${ }^{3}$ \\ ${ }^{1}$ Jodrell Bank Observatory, Department of Physics and Astronomy, \\ University of Manchester, Macclesfield, Cheshire SK11 9DL, UK \\ ${ }^{2}$ LUTh, Observatoire de Paris-Meudon, 92195 Meudon, France \\ ${ }^{3}$ Department of Applied Mathematics and Theoretical Physics, Centre for Mathematical Sciences, \\ University of Cambridge, Wilberforce Road, Cambridge CB3 OWA, UK
}

(Dated: November 25, 2018 - DRAFT)

\begin{abstract}
We discuss the linearized, gravitational self-interaction of a brane of arbitrary codimension in a spacetime of arbitrary dimension. We find that in the codimension two case the gravitational self-force is exactly zero for a Nambu-Goto equation of state, generalizing a previous result for a string in four dimensions. For the case of a 3-brane, this picks out the case of a six-dimensional brane-world model as having special properties which we discuss. In particular, we see that bare tension on the brane has no effect locally, suppressing the cosmological constant problem.
\end{abstract}

PACS numbers: $04.50,98.80$

The divergent self-force of a charged point particle, such as the electron, coupled to electromagnetism has been understood for many years. Its resolution via the inclusion of an ultra-violet (UV) cut-off, due to the finite radius of the particle, leads to a renormalization of the particle's mass and a suppression of the pole singularity at short distances (see, for example, [1])

This problem is not unique and, in fact, similar problems exist for any distributional source coupled to any kind of field in any spacetime dimension. An interesting case is that of a Nambu-Goto string coupled to linearized gravity. It has been shown [2, 3, 4] that the self-force, regularized in the UV by the core width of the string, $\epsilon$, and in the infra-red (IR) by the inter-string separation, $\Delta$, is exactly zero due to the fact that the induced linearized metric perturbation is orthogonal to the string worldsheet. This result can be shown to be true at all orders in perturbation theory, in the case of a static string [5].

A similar result can be deduced when the string in four dimensions is coupled to an axion field, represented by a 2 -form, and a dilaton, as well as linearized gravity $\underline{6}, 7,8$. predicted [9] in the context of $\mathcal{N}=1, D=10$ Supergravity, one can show that the combined self-interaction is zero; the dilaton contribution is negative, which cancels the positive contribution from the axion field.

It should be noted that the UV regularization of the self-field is not necessary in the codimension one case, the hypersurface, where the behaviour at the brane can be dealt with using junction conditions. The case of gravity can be dealt with exactly, at all orders, using the conditions often attributed to Israel [10] (although see ref. 11]). Similar lines of argument lead to the junction conditions at a surface in Maxwell's theory of electromagnetism [1].

The extension of these ideas to higher dimensions has become more relevant recently with the interest that has arisen in brane-world models. In these models, the mat- ter of the Standard Model of particle physics is confined to a four-dimensional subspace, or brane, of a higher-dimensional spacetime, often called the bulk. Two types of model have received particular attention: sixdimensional models with flat, compact extra dimensions, such as the Arkani-Hamed, Dimopoulos and Dvali (ADD) model [12], and five-dimensional models with warped extra dimensions, such as the Randall-Sundrum (RS) models [13]. These ideas were originally motivated by the notion of D-branes in M-Theory, and the desire to alleviate the weak hierarchy problem of UV quantum field theory (QFT). However, much subsequent work has focused on their gravitational properties.

Both models can be extended to higher dimensions. As we have discussed, the five-dimensional case has no UV divergence, so does not need to be regularized. When the extra dimensions are compact, the volume of the extra dimensions gives an effective IR cut-off scale. In the warped case, the curvature lengthscale of the bulk spacetime fulfils a similar role. In more general cases it is clear that some physical phenomena must provide either a UV cut-off (usually the thickness of the brane) or one in the IR (usually the distance between branes, or the background curvature length scale). In the codimension two case, one requires both since the self-field is proportional to $1 / r$ and the divergence of the self-energy is logarithmic.

One intriguing aspect of brane-worlds with two extra dimensions [14] is that the bare tension of the brane, which represents vacuum energy, does not appear to gravitate from the point of view of an observer on the brane; its effects only being felt in the bulk as a modification to the conical deficit angle. This can be thought of as a self-tuning model, suppressing the cosmological constant problem since the large variations in the vacuum energy expected due to, for example, cosmological phase transitions would not be experienced gravitationally by observers on the brane. 
As we have described the study of self-interactions finds applications in a wide range of research areas, from cosmic defects to superstring and M-theory. In this letter we discuss systematically the regularization of the gravitational self-force for extended objects with any codimension more than one, albeit at linearized order. Our results will also be relevant to the codimension one case, but as we have already noted, they are not completely necessary there. We find that, in the case of a Nambu-Goto brane the self-force takes a simple form and can be interpreted as a renormalization of the tension. In the codimension two case, this renormalization is exactly zero, extending the result for cosmic strings [2, 3, 4] to hyper-strings in arbitrary spacetime dimension. Our analysis allows for a general configuration of the brane and for background curvature on a scale greater than the effective width of the brane. It is, therefore, an extension of the self-tuning cosmological constant idea, in that previous work 14] has considered only symmetric, exact solutions in specific background spacetimes. We will consider the analogue of refs. [6, 7, , 8], which includes the effect of a dilaton and an antisymmetric form field, in a more detailed forthcoming paper.

We will consider a $p$-brane, with $(p+1)$-dimensional worldsheet, in an $n$-dimensional spacetime. The position of the brane will be given in terms of the spacetime coordinates $x^{\mu}$ by $x^{\mu}=X^{\mu}\left\{\sigma^{a}\right\}$, where $\sigma^{a}$ are internal worldsheet coordinates. The induced metric on the brane is then given by $\gamma_{a b}=g_{\mu \nu} \partial_{a} X^{\mu} \partial_{b} X^{\nu}$, and the background energy-momentum tensor, $\widehat{T}^{\mu \nu}$, due to that supported on the worldsheet, $\bar{T}^{\mu \nu}$, is

$$
\widehat{T}^{\mu \nu}\{x\}=\frac{1}{\sqrt{-g}} \int \bar{T}^{\mu \nu} \delta^{(n)}\left\{x-X\left\{\sigma^{a}\right\}\right\} \sqrt{-\gamma} \mathrm{d}^{p+1} \sigma .
$$

The first fundamental tensor of the brane and its orthogonal compliment can then be defined as $\eta^{\mu \nu}=$ $\gamma^{a b} \partial_{a} X^{\mu} \partial_{b} X^{\nu}$ and $\perp_{\mu \nu}=g_{\mu \nu}-\eta_{\mu \nu}$, respectively. These act as the projection operators tangential and orthogonal the worldsheet. The second fundamental tensor of the worldsheet and the extrinsic curvature vector are defined as

$$
K_{\mu \nu}{ }^{\rho}=\eta_{\mu}{ }^{\alpha} \eta_{\nu \beta} \nabla_{\alpha} \eta^{\beta \rho}, \quad K^{\rho}=g^{\mu \nu} K_{\mu \nu}{ }^{\rho} .
$$

This formulation in terms of background tensorial quantities has the advantage of avoiding the complications of then internal indices, $\sigma^{a}$. In the case of a codimension one brane, $\perp_{\mu \nu}=n_{\mu} n_{\nu}, K_{\mu \nu}=K_{\mu \nu}{ }^{\rho} n_{\rho}$ and $K^{\rho}=K n^{\rho}$ where $n_{\rho}$ is the unit normal covector to the brane, and $K_{\mu \nu}, K$ are the more familiar extrinsic curvature pseudotensor and scalar respectively. Note that we use the sign conventions of [15], whereas, some authors define the extrinsic curvature with the opposite sign.

We will perform our regularization calculation in a flat background spacetime, but it will also be valid in the case where the background is curved so long as the associated curvature scale is larger than the brane thickness. In the case of an Anti-de-Sitter (AdS) background this would require that the AdS length scale, $l \gg \epsilon$. Moreover, we also demand that the curvature scale of the brane be much larger than the brane thickness, i.e., $\sqrt{K^{\rho} K_{\rho}} \gg \epsilon$. This condition would not hold, for example, at a cusp in the brane worldsheet.

We consider a perturbation of the metric $g_{\mu \nu} \rightarrow$ $g_{\mu \nu}+h_{\mu \nu}$ with $\left|h_{\mu \nu}\right| \ll 1$, which, in an $n$-dimensional Minkowski spacetime, will satisfy the linearized Einstein equation

$$
\square h_{\mu \nu}=-2(n-2) \Omega^{[n-2]} \mathrm{G}\left(\widehat{T}_{\mu \nu}-\frac{1}{n-2} \widehat{T} g_{\mu \nu}\right),
$$

where $\square=\nabla_{\rho} \nabla^{\rho}$ is the wave operator defined by the unperturbed metric, $\mathrm{G}=M^{2-n}$, is the gravitational coupling constant appropriate to the background spacetime and $\Omega^{[n]}$ is the area of a unit $n$-sphere.

In order to perform the regularization, we make the split of the metric perturbation $h_{\mu \nu}=\hat{h}_{\mu \nu}+\tilde{h}_{\mu \nu}$, where $\hat{h}_{\mu \nu}$ is the singular contribution from the string and $\tilde{h}_{\mu \nu}$ is the (finite) remainder due to radiation backreaction and external effects. Defining the standard Green's function $\mathcal{G}\left\{x, X\left\{\sigma^{a}\right\}\right\}$ for the wave operator in Minkowski space, we find that

$h_{\mu \nu}\{x\}=\beta \int\left(\bar{T}_{\mu \nu}-\frac{1}{n-2} \bar{T} g_{\mu \nu}\right) \mathcal{G}\left\{x, X\left\{\sigma^{a}\right\}\right\} d^{p+1} \sigma$,

where $\beta=-2(n-2) \Omega^{[n-2]} \mathrm{G} / \Omega^{[n-1]}$. Using the standard form for the Green's function, this solution can be regularized, both in the UV and the IR, to give

$$
\hat{h}_{\mu \nu}=2 \mathrm{G}\left(\bar{T}_{\mu \nu}-\frac{1}{n-2} \bar{T} g_{\mu \nu}\right) F_{\{\Delta, \epsilon\}},
$$

where we have defined a regularization factor

$$
F_{\{\Delta, \epsilon\}}=\frac{\Omega^{[n-2]} \Omega^{[p]}}{\Omega^{[n-1]}} \int_{\epsilon}^{\Delta} s^{p+2-n} d s,
$$

to describe the dependence on the IR and UV cut-offs. At its simplest level this represents a hard cut-off in source density for $s<\epsilon$ and $s>\Delta$, but the cut-offs could easily be thought as effective, representing the envelope of a solution. The factor $F_{\{\Delta, \epsilon\}}$ encapsulates all of the dependence on the internal structure of the string and the effect of spacetime compactification or curvature on $\hat{h}_{\mu \nu}$. The regularization can be justified by a more rigorous calculation as described in [16].

The effective UV cut-off scale, $\epsilon$, is governed by the internal structure of the brane. Except in the codimension one case, the infinitely thin limit leads to a divergence, meaning that the profile of the brane is always important. The profile of the brane will remove the divergence associated with an infinitely thin source, thus generating an 
effective thickness, which we will assume is the same at every point on the worldsheet. For the domain wall case, such as the RS models [13], there is no UV divergence and the infinitely thin limit can be used.

The IR cut-off scale, $\Delta$, is necessary when considering branes of codimension one or two (domain walls and hyper-strings). The IR divergence can be removed in several ways, each of which will generate an effective value of $\Delta$. One such way is the usual Kaluza-Klein approach where one compactifies the extra dimensions on a circle or torus, the radius or volume of the internal space giving the effective cut-off scale. Another possibility is to consider an AdS bulk, as in the RS model, where the different form of the Green's function will mean that the corresponding integral does not have an IR divergence. The AdS lengthscale, $l$, will then give an effective cut-off, $\Delta \sim l$, allowing us to use the solution (5). Another possibility would be to consider a network of branes where the inter-brane separation would give an IR cut-off scale, as is usually assumed to be the case for $p=1, n=4$, i.e., cosmic strings.

One can determine the force acting on the brane by considering the variation of the brane component to the matter action

$$
\mathcal{S}=\int \overline{\mathcal{L}} \sqrt{-\gamma} \mathrm{d}^{p+1} \sigma
$$

Under the perturbation $g_{\mu \nu} \rightarrow g_{\mu \nu}+h_{\mu \nu}$, the first order change in the Lagrangian is given by

$$
\overline{\mathcal{L}}_{\text {eff }}=\overline{\mathcal{L}}+\frac{1}{4} h_{\rho \sigma} \bar{T}^{\rho \sigma},
$$

an extra factor of $1 / 2$ being the adjustment required to make sure the contributions are only counted once. Varying the action [2] shows us that the force on the brane is given by

$$
\bar{f}^{\mu}=\frac{1}{2} \bar{T}^{\nu \rho} \nabla^{\mu} h_{\nu \rho}-\nabla_{\nu}\left(\bar{T}^{\nu \rho} h_{\rho}{ }^{\mu}+\bar{C}^{\mu \nu \rho \sigma} h_{\rho \sigma}\right),
$$

where the hyper-Cauchy tensor is defined by

$$
\bar{C}^{\mu \nu \rho \sigma}=\frac{1}{\sqrt{-\gamma}} \frac{\delta}{\delta g_{\mu \nu}}\left(\sqrt{-\gamma} \bar{T}^{\rho \sigma}\right)
$$

which is a relativistic version of the Cauchy elasticity tensor.

In order the evaluate the regularized force from (9), we need to compute the regularized version of the gradient $\nabla_{\rho} h_{\mu \nu}$. We will do this using the formula derived in ref. 16] for scalar field $\phi$,

$$
\widehat{\nabla_{\rho} \phi}=\eta_{\rho}{ }^{\sigma} \nabla_{\sigma} \hat{\phi}+\frac{1}{2} K_{\rho} \hat{\phi},
$$

can applied to each of the component of $h_{\mu \nu}$ since we are considering linearized interactions. This formula applies when $p>0$ and when the codimension is greater than one. The factor of $1 / 2$ in the second term should be replaced by $(p-1) / 2 p$ in the codimension one case. The first term in this formula is just the derivative tangent to the brane, which is all one would have in the case of a straight brane. The effect of the curvature is in the second term, which accounts for the change in orientation of the planes normal to the brane when it is curved within the bulk.

For a Nambu-Goto type brane, the Lagrangian, energy-momentum tensor and hyper-Cauchy tensor are given by $\overline{\mathcal{L}}=-\lambda=-m^{p+1}, \bar{T}^{\mu \nu}=-\lambda \eta^{\mu \nu}$ and

$$
C^{\mu \nu \rho \sigma}=\lambda\left(\eta^{\mu(\rho} \eta^{\sigma) \nu}-\frac{1}{2} \eta^{\mu \nu} \eta^{\rho \sigma}\right)
$$

where $m$ is a fixed mass scale and $\lambda$ is the tension of the brane. The singular part of the metric perturbation is given by

$$
\hat{h}_{\mu \nu}=2 \lambda \mathrm{G} F_{\{\Delta, \epsilon\}}\left(\frac{p+3-n}{n-2} \eta_{\mu \nu}+\frac{p+1}{n-2} \perp_{\mu \nu}\right),
$$

which, in the codimension two case where $p=n-3$, will be purely orthogonal to the worldsheet.

If one regularizes the force (9), using the relation (11) for the regularized gradient and the solution (13), one can deduce that the linearized, gravitational self-force is given by

$$
\hat{f}^{\mu}=\frac{(p+1)(p+3-n)}{2(n-2)} \lambda^{2} \mathrm{G} F_{\{\Delta, \epsilon\}} K^{\mu},
$$

for a brane of codimension greater than one. In the codimension one case, there will be an additional factor of $(p+1) / p$. The force is in the direction of the extrinsic curvature vector, $K^{\rho}$, which is normal to the brane worldsheet. This can be interpreted as a renormalization of the tension of the brane

$$
\frac{\lambda_{\mathrm{eff}}}{\lambda}=1-\frac{(p+1)(p+3-n)}{2(n-2)} \lambda \mathrm{G} F_{\{\Delta, \epsilon\}} .
$$

This renormalization represents a correction to the Lagrangian $\overline{\mathcal{L}}$ of the matter supported on the brane, providing a term which looks like an effective cosmological constant on the brane. It is obvious that this force, and hence the renormalization, will vanish when $p=n-3$, generalizing the result previously derived for cosmic strings in four-dimensions [3, 4].

One can see this in much more simple terms, if one considers the action renormalization, that is, if one substitutes (13) into (8); (15) can be re-derived very easily by using the fact that $\eta_{\mu \nu} \eta^{\mu \nu}=p+1$ and one can then see directly that if $\hat{h}_{\mu \nu} \propto \perp_{\mu \nu}$, as is the case when $p=n-3$, then not only is the self-force zero, but so is the action renormalization.

For a brane-world, $p=3$, and this special case requires $n=6$. This phenomena has been pointed out recently by 
several authors [14] who have studied explicit solutions for specific formulations of six-dimensional brane-worlds. Here, we have generalized this result to arbitrary, nonstatic configurations at linearized order. One can see that for a general surface energy momentum tensor $\bar{T}^{\mu \nu}$, the action renormalization is given by

$$
\mathcal{L}_{\text {eff }}=\mathcal{L}+\frac{1}{2} \mathrm{G} F_{\{\Delta, \epsilon\}}\left(\bar{T}^{\mu \nu} \bar{T}_{\mu \nu}-\frac{1}{n-2} \bar{T}^{2}\right) .
$$

Some of these authors have suggested that this could have implications for the observed gravitational effects of vacuum energy in these models since the vacuum energy can be thought of as being the origin of the bare tension of the brane-world. Effective QFTs predict that the energy scale associated with the vacuum could be as large as the Planck mass, $M_{\mathrm{pl}}$, making it discrepant by a factor of the order $10^{120}$ with the upper bounds from observation. If $n=6$ and $p=3$ then our calculation has a simple, but elegant resolution to this problem; the bare tension only gravitates in the direction orthogonal to the brane-world, that is, $h_{\mu \nu} \propto \perp_{\mu \nu}$, the gravitational selfforce is zero and the renormalized action has no constant contribution. Hence, the effective cosmological constant as experienced by observers on the brane would be zero, providing a self-tuning mechanism for the cosmological constant. This establishes a link between the self-tuning phenomena at work here and the self-force.

We should note that there are some obvious problems associated with this self-tuning mechanism, not least the fact that there is evidence to suggest that the cosmological constant is non-zero [17]. Moreover, observations of the angular power spectrum of anisotropies in the cosmic microwave background [18] suggest that inflation is ultimate their origin. Since both require some kind of acceleration, albeit from a scalar field in the case of inflation, if this scenario were correct then this acceleration could not be due to any kind of brane-based effect since all vacuum energy can do is modify the bulk solution and can have no effects on the brane.

One resolution of this would be to generate accelerated expansion from bulk effects. A bulk cosmological constant will gravitate and give an effective cosmological constant as seen by a brane-based observer. Similarly, accelerated expansion could be driven by a bulk scalar field. Of course, one still has a cosmological constant problem in the bulk: how to determine what mechanism fixes this to be small. Furthermore, reheating after bulkinduced inflation could be problematic if the inflaton is not formed from brane-based matter.

To summarize: the main result of this paper is the generalization of the non-divergence of the self-force of a Nambu-Goto cosmic string to a corresponding hyperstring in arbitrary dimensions under the assumption that the solution is regularized by some physical phenomena in the UV and the IR. In fact, we have derived a gen- eral formula for the linearized gravitational self-force in arbitrary co-dimension and the corresponding renormalization of the bare tension. We have pointed out the links with recent work on attempts to self-tune the cosmological constant in $6 \mathrm{D}$ brane-world models. An analysis using a similar method to this but considering the gravitational interaction of observable matter supported on the brane, in the usual brane-world limit where it is small compared to the bare tension, would give a much firmer foundation to these ideas.

RAB is supported by PPARC and AM is supported by Emmanuel College.

[1] J. D. Jackson Classical Electrodynamics (1962) John Wiley and Sons Inc.

[2] R. A. Battye and B. Carter (1995) Phys. Lett. B 35729.

[3] B. Carter and R. A. Battye (1998) Phys. Lett. B 43049.

[4] A. Buonanno and T. Damour (1998) Phys. Lett. B 432 51.

[5] G. W. Gibbons (1999) Class. Quant. Grav. 16: 1471.

[6] E. Copeland, D. Haws and M. Hindmarsh (1990) Phys. Rev. D 42726.

[7] A. Dabholkar, G. W. Gibbons, J. A. Harvey and F. RuizRuiz (1990) Nucl. Phys. B 34033.

[8] B. Carter (1999) Int. J. Theor. Phys. 382779.

[9] A. Dabholkar and J. A. Harvey (1989) Phys. Rev. Lett. 63478.

[10] W. Israel (1966) Nuovo Cimento B, 441 erratum ibid. 48: 463.

[11] K. Lanczos (1922) Phys. Z. 23 539. K. Lanczos (1924) Ann. Phys. Lpz. 74 518. N. Sen (1924) Ann. Phys. Lpz. 73 365. G. Darmois (1927) Mémorial des Sciences Mathématique XXV. C. W. Misner and D. H. Sharp (1964) Phys. Rev. B 136571.

[12] N. Arkani-Hamed, S. Dimopoulos and G. Dvali (1998) Phys. Lett. B 429 263. I. Antoniadis, N. Arkani-Hamed, S. Dimopoulos and G. Dvali (1998) Phys. Lett. B 436 257.

[13] L. Randall and R. Sundrum (1999) Phys. Rev. Lett. 83 3370. L. Randall and R. Sundrum (1999) Phys. Rev. Lett. 834690.

[14] A. G. Cohen and D. B. Kaplan (1999) Phys. Lett. B 470 52. T. Gherghetta and M. Shaposhnikov (2000) Phys. Rev. Lett. 85 240. P. Kanti, R. Madden and K. A. Olive (2001) Phys. Rev. D 64 044021. S. M. Carroll and M. M. Guica (2003) hep-th/0302067 Y. Aghababaie, C. P. Burgess, S. L. Parameswaran and F. Quevedo (2003) hep-th/0304256. I. Navarro (2003) Class. Quant. Grav. 20 3603. H.-P. Nilles, A. Papazoglou and G. Tasinato (2003) hep-th/0309042 . J. M. Cline, J. Descheneau, M. Giovannini and J. Vinet (2003) JHEP 0306048.

[15] C. W. Misner, K. S. Thorne and J. A. Wheeler, Gravitation (1973) W. H. Freeman and Co.

[16] B. Carter, R. A. Battye and J.-P. Uzan (2003) Commun. Math. Phys. 235289.

[17] S. Perlmutter et al. (1999) Astrophys. J. 517: 565-586.

[18] D. N. Spergel et al. (2003) Astrophys. J. Suppl. 148: 175. H. V. Peiris et al. (2003) Astrophys. J. Suppl. 148: 213. 